\title{
A Comparative Analysis on Korean Medical and Western Medical Service Usage Tendency of Rotator Cuff Surgery Patients - Using HIRA's Patients Sample Data
}

\author{
Hyun-jin Khang ${ }^{1}$, Hye-Yoon Lee ${ }^{2}$, Se-Yeon Lee ${ }^{2}$, NamKwen Kim ${ }^{2 *}$, YunKyung Song ${ }^{1}$ \\ ${ }^{1}$ Department of Korean Medicine, Graduate School of Gachon University \\ ${ }^{2}$ School of Korean Medicine, Pusan National University
}

\begin{abstract}
Objectives: To lay the foundation for future research into Korean Medicine treatment for Rotator Cuff repair surgery patients by analyzing Korean Medical and Western Medical service utilization and treatment duration.

Methods: Data sampling was performed on 2015's HIRA patient data (confidence level of 97\%) to analyze patients' Korean Medical and Western Medical service usage tendency. Sampled groups were divided into two groups: i) Patients who completed their treatment within five months after the rotator cuff surgery (termination group), ii) Patients who were treated for more than five months after the surgery (continuation group). Then the patients' Korean Medical and Western Medical service usage tendency was investigated and information of these patients are arranged. Results: Out of 1,453,486 patients who were gathered for sampling, 2,461 patients in total had gone through rotator cuff repair surgery. The termination group had 517 patients and the continuation group had 541 patients. The proportion of patients who visited a Korean Medicine clinic was lower in the termination group than the continuation group.

Conclusion: The continuation group received more treatments (both in Western Medicine and Korean Medicine) and spent more on medical expenses compared to the termination group. Further research is highly recommended for more efficient Western Medicine and Korean Medicine treatments and reduced medical expenditure.
\end{abstract}

$\overline{\text { Key Words }}$ : Rotator Cuff repair, Acromioplasty, Shoulder

\section{Introduction}

Recently the importance of proper postoperative rehabilitation and care is being emphasized more with an increase in musculoskeletal surgery due to sports injuries from the general public and athletes, in addition to surgery due to degenerative changes. (1) The 2008 U.S. statistics show that more than 17 million patients suffer from rotator cuff tear, which is the most frequent condition among shoulder joint diseases and the most likely condition to be operated on.(2) However, many patients suffer from postoperative symptoms such as pain or stiffness. Therefore, clinically appropriate postoperative rehabilitation is critical and should focus on reducing pain that restricts

\footnotetext{
- Received : 9 September $2021 \quad$ - Revised : 24 October 2021 Accepted : 8 November 2021

- Correspondence to : NamKwen Kim

School of Korean Medicine, Pusan National University

Tel : +82-55-360-5947 E-mail : drkim@pusan.ac.kr Fax.+82-55-360-5906

- This study was funded by the Ministry of Health and Welfare. Health and Medical Technology of Korea Health Industry Development Institute R\&D project support (Project unique number: HB16C0011)
} 
movements, overcoming the R.O.M. (Range of Motion) restriction due to anatomical changes, and gradually recovering muscle strength. There are case studies on the rehabilitative treatment after rotator cuff surgery, but the research remains insufficient (3).

The causes of rotator cuff tear vary from not only degenerative changes due to aging but also problems in blood circulation and epidemiological causes. Recently, an increase in traumatic incidents due to outdoor activities has contributed to the cause as well (4).

When these causes result in rotator cuff tear, the treatment depends on the severity of the symptoms. in the case of a full tear, surgical treatment is considered from the beginning. And for a partial tear, non-surgical treatments are performed for about a year, and if there is no improvement then surgical treatment is considered (5). If surgery is performed due to a full or partial tear, various aftereffects such as restriction of movement may occur. Therefore, proper postoperative treatment should be given to help the patient return to daily life. Shin et al. (6) conducted a study on whether the degree of rotator cuff tear affected the degree of recovery after surgery. They found the muscle strength after the surgery was weaker when the degree of tear was bigger, but there was no difference in the recovery in terms of pain felt by patients. This suggests that the stage of recovery may differ depending on the degree of preoperative tear. And although the surgery of the tear itself may be successful, the postoperative treatment may be inadequate, resulting in pain or stiffness after the surgery. (1)
Korean Medicine (KM) utilizes herbal medicine, acupuncture, physical therapy, in addition to pharmacopuncture and Chuna therapy to rehabilitate the patients after musculoskeletal surgeries (7). Kim, et al (8) reported no significant difference in terms of pain control in the treatment group that only used KM rehabilitation therapy and the treatment group that used combination therapy with an analgesic. However, clinical practice guideline in applying $\mathrm{KM}$ therapy postoperatively does not exist yet (9). In addition, there have been no studies using the health insurance claims data on rotator cuff tear, including the analysis of each invoice, principal diagnosis per each surgery, and the medical service usage behavior of patients depending on their use of Western Medical services or Korean Medical services. This study is thought to reflect the real-world situation most closely by analyzing the National Patient Sample Data(NPS) provided by the Health Insurance Review and Assessment Service(HIRA) in 2015.

Since Western Medicine (WM) was introduced to Korea more than 100 years ago, KM and WM have coexisted parallelly within a dual medical system in Korea. Although the two medical disciplines have improved the health of the population with their respective strengths, it has been pointed out that gaps in a dualized medical system and a lack of cooperation have caused inefficiencies in the medical system. To solve these problems and improve the quality of health care services, multidisciplinary cooperation between KM and WM (hereinafter referred to as KM-WM Collaboration) has been actively discussed (10). 
In response, we intend to investigate the demand for $\mathrm{KM}$ rehabilitation treatment of patients suffering from postoperative symptoms by centrally analyzing the usage of $\mathrm{KM}$ rehabilitation treatment among patients who underwent western rehabilitation after operate, and the need for systematization of $\mathrm{KM}$ rehabilitation treatment after shoulder surgery.

To look at some previous research utilizing big data on KM, Korea Institute of Oriental Medicine (KIOM) has developed a questionnaire for a standardized diagnosis of Sasang (four types) constitutional medicine and created a Sasang constitution information bank by collecting diagnostic results from clinical experts (11). Moreover, in 2016 KIOM created Korean Medicine Data Center by compiling more databases including further clinical data on constitution types, sleep, pain, cold-heat pattern differentiation, body type measurement data, voice data, facial information, blood tests, and DNA. And it made the data available to anyone who needed it (12). Recently, there have been a growing number of studies on the medical service usage behavior of patients seeking KM treatments $(13,14)$. Also, the usage of acupuncture and electric acupuncture on patients after musculoskeletal surgeries has been studied utilizing big data from the Health Insurance Review and Assessment Institute (15). In addition, more research is being conducted abroad in applying acupuncture therapy to reduce opioid use after surgeries, thereby revealing the efficacy of acupuncture for postoperative pain (16). Therefore, we deemed it sufficiently necessary to carry out a study to statistically investigate the current clinical situation in Korea regarding the use of acupuncture treatment for postoperative pain. Furthermore, Song et al. (17) proposed a manual for KM-WM collaborative rehabilitation treatment after effective shoulder surgery. And Lee et al.'s research demonstrated that indexes such as NRS (Numerical Rating Scale) and SPADI (Shoulder Pain and Disability Index) improved significantly after receiving KM-WM collaborative rehabilitation treatment for rotator cuff tear(18).

However, these prior studies lack objectivity and detailed analysis according to disease type because they have not studied big data enough on a specific disease like rotator cuff tear nor utilized objective indicators such as data from the Health Insurance Review and Assessment Service.

Accordingly, we analyzed the general characteristics and $\mathrm{KM} / \mathrm{WM}$ service usage behavior of rotator cuff tear patients by using the National Patients Sample data from the Health Insurance Review \& Assessment Service (HIRA_NPS) in 2015. And regarding the patients' KM service use, we looked at when they first visited a KM clinic and investigated the relationship between the KM use and their treatment results. Finally, we planned for this study to lay the foundation for future research into $\mathrm{KM}$ treatment on rotator cuff tear patients.

\section{Research and analysis methodology}

\section{Research data}

The data of this study are the $3 \%$ sample that we extracted out of the total patients that 
received national healthcare insurance coverage between Jan. 1 and Dec. 31, 2015. All the National Patient Sample Data (HIRA_NPS) are available for public use from the Health Insurance Review \& Assessment Service. In 2015 , a total of $1,453,486$ patients were registered under the musculoskeletal disease code. Utilizing data from multiple years limits us from tracking the patients with surgery codes, so we set the scope to the one-year data in 2015 to reduce the error in tracking. In addition, to secure a follow-up period of up to six months after surgery, we targeted patients who received surgery before June 1, 2015.

The data consist of demographic characteristics such as gender, average age, and variables such as disease registration codes and insurance-covered items from each medical institution. And statistically sampled secondary data were provided after removing specifiable information about individuals and corporations from the raw data.

The data used in this study are public data provided by the government under 'the Public Data Provision and Activation Act (19)', which are appropriate as research data because the data reflect the actual medical environment while saving the cost, time, and effort required for sampling.

\section{Patients with rotator cuff surgery}

In order to extract information relevant to this research from a national patient sample, we selected rotator cuff surgery-related disease codes based on the International Classification of Diseases-10-Procedural Coding System (ICD-10 -PCD), and selected disease codes for rotator cuff surgery and damage or shoulder related disease or rotator cuff related disease based on the Korean Standard Classification of Disases-7 (KCD-7). Then we determined the frequency of principal diagnosis based on the surgery, as well as analyzing an intersection group that satisfied both the rotator cuff surgery disease code and the rotator cuff tear disease code when tracking the patients' $\mathrm{KM}$ and WM clinic usage behavior after receiving surgery.

In addition, we wanted to know the differences between the patients with rotator cuff surgery and other patients. Therefore, we compared the socio-anthropological characteristics of the rotator cuff surgery patients with the entire sample data other than the target subjects of the research.

KCD-7 codes are systematically classified based on the similarity of diseases, employing four-unit codes (A00.0) rather than the less specific three-unit code (A00). We originally aimed to analyze the data with four-unit codes, such as M75.1 (rotator cuff tear), however, this turned out to be impossible, so we analyzed using three-unit codes.

\section{Classification of medical institutions and insurance benefits}

Using medical institution type codes, KM hospitals and KM local clinics were classified as $\mathrm{KM}$ institutions, and other medical providers (WM local clinics, general hospitals, tertiary hospitals, long-term care hospitals, psychiatric long-term care hospitals, public health centers, health subcenters, community healthcare providers, mother and child health centers and pharmacies) were classified as WM institutions. The medical 
institution type statements were categorized based on institution type codes and data format code variables. KM statements were limited to $\mathrm{KM}$ outpatient and $\mathrm{KM}$ inpatient codes, and other classification codes were categorized under WM statements. The category of national health insurance, Medial Aid Program, and veterans' healthcare were classified according to insurance -covered items on the statement. The total number of statements was 27,546,105.

\section{Analysis of medical expenses}

Based on the insurance fee spent on each medical service (including KM and WM) after a rotator cuff surgery, we analyzed and compared the insurance claims data and medical service usage pattern of WM using group and KM using group by calculating the average amount of insurance payment per treatment for each group.

In addition, we analyzed how each patient paid medical expenses by dividing the insurance provider type into national health insurance, Medical Aid Program, and veterans' healthcare.

\section{Continuation of treatment after rotator cuff surgery}

To track whether patients who received rotator cuff surgery complete treatment at an appropriate time or continue on with treatments, we targeted those patients who received the surgery before June 1, 2015, and set the maximum tracking period as six months. And they were divided into treatment continuation group and treatment termination group based on whether they continued treatment or not.

The continuation group was defined as those who underwent re-operation after the first surgery or who were treated for more than five months under the same principal diagnosis. If the patient underwent re-operation within 14 days after the first surgery, then they were tracked from the date of the re-operation.

The termination group was defined as those whose visits to medical institutions have not been confirmed for more than one month under the same principal diagnosis five months after the surgery. We set the recovery period as five months based on the study that the average time of discovering a re-tear on the MRI after rotator cuff repair surgery was 5.3 months (20).

\section{Statistical analysis}

Statistical analysis was performed to test the homogeneity between the rotator cuff surgery group (target group) and the rest of the population excluding the target group. For discrete variables, we performed Fisher's exact test if the cells had an expected frequency of 5 were $50 \%$ or higher, and Chi-square test if the cells had an expected frequency of $50 \%$ or lower. For continuous variables, we performed t-test. After processing the variables in the data according to the analysis based on patients and statements, we analyzed the demographic characteristics and descriptive statistics on medical expenses and medical service usage behavior by KM and WM treatment groups. Sample data were divided into national health insurance, Medical Aid Program, and veterans' healthcare to investigate the distribution of the type of insurance being used by patients. All statistical analyses were conducted using SAS at a significance level of 
0.05 with a two-tailed test.

\section{Results}

\section{General characteristics of rotator cuff} surgery patients and the rest of the population in the sample data excluding the target patients in HIRA_NPS in 2015

According to the 2015 national patient sample data (HIRA_NPS) from the Health Insurance Review \& Assessment Service, the sample data population excluding the target group consisted of 706,964 men $(48.72 \%)$ and 744,061 women (51.28\%). The target group included the 2,461 patients who received rotator cuff surgeries, and of those 2,461 were men (50.3\%), and 1,223 were women $(49.7 \%)$.

In addition, out of the sample population excluding the target group, 97.04\% was covered by the national health insurance, $2.92 \%$ was covered by the Medical Aid Program 2.92\%, and $0.04 \%$ was covered by veterans' healthcare. Meanwhile, out of the patients who received rotator cuff surgery, $97.24 \%$ were covered by national health insurance, $2.6 \%$ by the Medical Aid Program, and $0.16 \%$ by the veterans' healthcare.

The average age of those who received rotator cuff surgery was 54.91 years, and that of those who were diagnosed principally with rotator cuff tear without receiving rotator cuff surgery was 39.76 years. Therefore, the age of the subjects who underwent surgery is 15.15 years older than the group who did not.

In addition, we compared the number of WM and $\mathrm{KM}$ treatments received by the patients who had the surgery and those who did not. Those who underwent surgery received WM treatments 43.40 times and KM treatments 5.26 times. The group with no surgery received WM treatments 16.62 times and $\mathrm{KM}$ treatments 2.28 times. (Table 1)

Finally, the medical expenses for the sample population excluding the target averaged 1,033,411 KRW during the year 2015. And the target group spent an average of 4,626,323 KRW including the cost of surgery during that same year. We additionally examined the cost of rotator cuff surgery and the average cost per visit for $\mathrm{KM}$ and $\mathrm{WM}$ treatments. After analyzing 2,535 rotator cuff surgery insurance statements, the average cost for a rotator cuff surgery was found to be 2,474,634 KRW. The average cost of WM treatment excluding the surgery was about 33,219 KRW per session, calculated from 41,563 WM treatment insurance statements. And from 3,240 KM treatment insurance statements, the average medical cost of KM treatment was about 25,892 KRW per session. (Table 2)

\section{Frequency analysis of rotator cuff tear patients and frequency analysis of the number of operations}

Based on the number of principal diagnoses registered as surgeries, we analyzed the operation frequency of the patient group who underwent a rotator cuff surgery. 768 (29.99\%) was registered as N0935 (Acromioplasty), 1,295 (50.57\%) was registered as N0936 (Acromioplasty and Repair of Ruptured Shoulder Rotator Cuff-Primary Repair), 374 (14.6\%) was registered as N0937 
(Acromioplasty and Repair of Ruptured Shoulder Rotator Cuff-with Myoplasty and Tendoplasty), and $124(4.84 \%)$ was registered as N0938 (Acromioplasty and Repair of Ruptured Shoulder Rotator Cuff-Complex).

Based on principal diagnoses registered as surgeries, we analyzed KCD used in each principal diagnose. In N0935, 617(80.34\%) was registered as M75(Shoulder lesions), 84(10.94\%) as S46(Injury of muscle and tendon at shoulder and upper arm level), 8(1.04\%) as S43(Dislocation, sprain and strain of joints and ligaments of shoulder girdle). In N0936, 834(64.4\%) as M75, 338(26.1\%) as S46, 43(1.32\%) as S43. In N0937, 239(63.9\%)

Table 1. Characteristics of the 2015 HIRA_NPS Patients with Surgery \& Others

\begin{tabular}{|c|c|c|c|c|}
\hline \multirow[b]{2}{*}{ Variables } & \multicolumn{3}{|c|}{ Patients } & \multirow[b]{2}{*}{ p-value ${ }^{*}$} \\
\hline & $\begin{array}{c}\text { All } \\
\mathrm{N}^{*}(\%)\end{array}$ & $\begin{array}{c}\text { Surgery } \\
\mathrm{N}(\%)\end{array}$ & $\begin{array}{l}\text { Others }^{*} \\
\mathrm{~N}(\%)\end{array}$ & \\
\hline \multicolumn{5}{|l|}{ Sex } \\
\hline Male & $708,202(48.72)$ & $1,238(50.3)$ & $706,964(48.72)$ & \multirow{3}{*}{0.1165} \\
\hline Female & $745,284(51.28)$ & $1,223(49.7)$ & $744,061(51.28)$ & \\
\hline Total & $1,453,486(100)$ & $2,461(100)$ & $1,451,025(100)$ & \\
\hline \multicolumn{5}{|l|}{ Insurance } \\
\hline N.H.I* & $1,410,481(97.04)$ & $2,393(97.24)$ & $1,408,088(97.04)$ & \multirow{4}{*}{0.0061} \\
\hline M.A.P & $42,427(2.92)$ & $64(2.6)$ & $42,363(2.92)$ & \\
\hline V.H.C & $578(0.04)$ & $4(0.16)$ & $574(0.04)$ & \\
\hline \multirow[t]{2}{*}{ Total } & $1,453,486(99.9)$ & $2,461(100)$ & $1,451,025$ (99.9) & \\
\hline & Mean \pm SD & Mean \pm SD & Mean \pm SD & \\
\hline Age & $39.79 \pm 21.48$ & $54.91 \pm 11.98$ & $39.76 \pm 21.42$ & $<0.0001$ \\
\hline $\begin{array}{l}\text { West Med. Use } \\
\text { (frequency) }\end{array}$ & $16.67 \pm 20.35$ & $43.40 \pm 30.57$ & $16.62 \pm 20.30$ & $<0.0001$ \\
\hline $\begin{array}{l}\text { Korean Med. Use } \\
\text { (frequency) }\end{array}$ & $2.28 \pm 8.63$ & $5.26 \pm 10.87$ & $2.28 \pm 8.62$ & $<0.0001$ \\
\hline Medical Cost (W) & $\begin{array}{c}1,039,494.41 \\
\pm 3,866,982.55\end{array}$ & $\begin{array}{c}4,626,323 \\
\pm 4,477,405\end{array}$ & $\begin{array}{c}1,033,411 \\
\pm 3,863,039\end{array}$ & $<0.0001$ \\
\hline
\end{tabular}

Table 2. Medical cost per session of surgery, western (except surgery) \& Korean medical institutions based on claims in the surgery group

\begin{tabular}{lccc}
\hline Variables & $\mathrm{N}^{*}$ & Mean \pm SD & Min $\sim$ Max \\
\hline Surgery cost & 2,535 & $2,474,634 \pm 1,133,743$ & $81,010 \sim 31,258,870$ \\
Western Med. cost except surgery $^{*}$ & 41,563 & $33,218.64 \pm 116,349.4$ & $0 \sim 6,545,650$ \\
Korean Med. cost $^{*}$ & 3,240 & $25,892.04 \pm 60,034.8$ & $0 \sim 1,519,200$ \\
\hline
\end{tabular}

${ }^{*} \mathrm{~N}$ : Number of total visits,

Western Med. cost except surgery: cost which paid for treatment in Western medical institution except surgery,

Korean Med. cost: cost which paid for treatment in Korean medical institution 
as $\mathrm{M} 75,120(32.09 \%)$ as $\mathrm{S} 46,6(1.6 \%)$ as $\mathrm{S} 43$. In 0938, 75(60.48\%) as M75, 44(35.78\%) as S46, 2(1.61\%) as M24(Other specific joint derangements) and S43, respectively. (Table 3, 4, $5,6)$

To find out how many patients underwent multiple surgeries and received the surgery code multiple times, we analyzed of the frequency of rotator cuff surgery based on the number of patients. Out of the 2,461 surgeries, 2,389 patients $(97.07 \%)$ received the surgery once, 70 patients $(2.84 \%)$ received it twice, and two patients $(0.08 \%)$ received it three times.

\section{The correlation between the length of the postoperative period and the patients' first visit to a KM institution} Looking into the patients' first visit to a KM

Table 3. Frequent disease code analysis in N0935

\begin{tabular}{llr}
\hline Code & Disease & $\mathrm{N}^{*}(\%)$ \\
\hline M75 & Shoulder lesions & $617(80.34)$ \\
S46 & Injury of muscle and tendon at shoulder and upper arm level & $84(10.94)$ \\
S43 & Dislocation, sprain and strain of joints and ligaments of shoulder girdle & $8(1.04)$ \\
M62 & Other disorders of muscle & $6(0.78)$ \\
M65 & Synovitis and tenosynovitis & $6(0.78)$ \\
M13 & Other arthritis & $5(0.65)$ \\
M19 & Other arthrosis & $5(0.65)$ \\
S49 & Traumatic amputation of shoulder and upper arm & $5(0.65)$ \\
S42 & Fracture of shoulder and upper arm & $4(0.52)$ \\
M00 & Pyogenic arthritis & $3(0.39)$ \\
Extra Diseases & & $25(3.25)$ \\
\hline
\end{tabular}

${ }^{\text {NN}}$ :Number of used codes, Extra Diseases:Involving all other codes used 1 or 2 times

Table 4. Frequent disease code analysis in N0936

\begin{tabular}{llr}
\hline Code & Disease & $\mathrm{N}^{*}(\%)$ \\
\hline M75 & Shoulder lesions & $834(64.4)$ \\
S46 & Injury of muscle and tendon at shoulder and upper arm level & $338(26.1)$ \\
S43 & Dislocation, sprain and strain of joints and ligaments of shoulder girdle & $43(3.32)$ \\
M24 & Other specific joint derangements & $25(1.93)$ \\
M25 & Other joint disorders, NEC & $16(1.24)$ \\
M65 & Synovitis and tenosynovitis & $5(0.39)$ \\
M62 & Other disorders of muscle & $4(0.31)$ \\
M66 & Spontaneous rupture of synovium and tendon & $4(0.31)$ \\
M51 & Other intervertebral disc disorders & $3(0.23)$ \\
S42 & Fracture of shoulder and upper arm & $3(0.23)$ \\
Extra Diseases & & Total \\
\end{tabular}

${ }^{\text {NN}}$ :Number of used codes, Extra Diseases:Involving all other codes used 1 or 2 times 
institution after a rotator cuff surgery, we found that about $25 \%$ of all patients who received both $\mathrm{KM}$ and $\mathrm{WM}$ treatments visited a $\mathrm{KM}$ institution within 33 days after the surgery, and about 50\% started receiving KM treatments within 73 days. According to a hierarchical logistic regression of postoperative tear, we analyzed the 1058 cases that received $\mathrm{WM}$ and/or $\mathrm{KM}$ treatments after the surgery out of the 2461 patients that underwent a rotator cuff surgery. The Odds Ratio that includes all predictors of the period when $25 \%$ of the subjects received KM treatment (Model 1), the period when 50\% received KM treatment (Model 2), and six months after the surgery (Model 3) was calculated to be 2.16. The results demonstrate that as the postoperative care period increased, the number of $\mathrm{KM}$ treatments increased as well. (Table 7)

\section{Analysis of medical institution usage after a rotator cuff surgery}

According to a six-month follow-up of patients who underwent a rotator cuff surgery before June 1, 2015, out of the 933 patients who received WM treatments (WM group), 493 $(52.84 \%)$ were men and $440(47.16 \%)$ were women. And out of the 125 patients that received $\mathrm{KM}$ treatments (KM group), (34.4\%) were men and $82(65.6 \%)$ were women. The difference was that $5.68 \%$ more men received WM treatments compared to women, and $31.2 \%$ more women received $\mathrm{KR}$ treatments compared to men. However, the KM group includes those who received both $\mathrm{KM}$ and WM treatments due to $\mathrm{WM}$ treatments received during the preparation before and the rehabilitation after the surgery.

In addition, the average age of the WM group was 54.40 years, while the average age of $\mathrm{KM}$

Table 5. Frequent disease code analysis in N0937

\begin{tabular}{llr}
\hline Code & Disease & $N^{*}(\%)$ \\
\hline M75 & Shoulder lesions & $239(63.9)$ \\
S46 & Injury of muscle and tendon at shoulder and upper arm level & $120(32.09)$ \\
S43 & Dislocation, sprain and strain of joints and ligaments of shoulder girdle & $6(1.6)$ \\
& & Extra Diseases
\end{tabular}

${ }^{\text {*N}} \mathrm{N}$ :Number of used codes, Extra Diseases:Involving all other codes used 1 or 2 times

Table 6. Frequent disease code analysis in N0938

\begin{tabular}{llr}
\hline Code & Disease & $\mathrm{N}^{*}(\%)$ \\
\hline M75 & Shoulder lesions & $75(60.48)$ \\
S46 & Injury of muscle and tendon at shoulder and upper arm level & $44(35.48)$ \\
M24 & Other specific joint derangements & $2(1.61)$ \\
S43 & Dislocation, sprain and strain of joints and ligaments of shoulder girdle & $2(1.61)$ \\
M19 & Other arthrosis & $1(0.81)$ \\
\hline
\end{tabular}

${ }^{*} \mathrm{~N}$ :Number of used codes 
group was 58.96 years, showing that average age of the KM group was 4.56 years older.

In terms of the type of insurance coverage of the WM group, the national health insurance accounted for $97.96 \%$, and Medical Aid Program and veterans' healthcare accounted for $2.04 \%$ of the health insurance. Meanwhile for the KM group, the national health insurance accounted for 97.6\%, and Medical Aid Program and veterans' healthcare accounted for $2.4 \%$ of the

Table 7. Logistic Regression Analysis of continuing treatment on Korean medical treatment in rotator cuff surgery patients $(\mathrm{N}=1,058)$

\begin{tabular}{|c|c|c|c|}
\hline \multirow{2}{*}{ Co-variables } & Model 1 & Model 2 & Model 3 \\
\hline & $\mathrm{OR}(95 \% \mathrm{Cl})$ & $\mathrm{OR}(95 \% \mathrm{Cl})$ & $\mathrm{OR}(95 \% \mathrm{Cl})$ \\
\hline Treatment Continue & $\begin{array}{c}2.75 \\
(1.82 \sim 4.15)\end{array}$ & $\begin{array}{c}2.47 \\
(1.63 \sim 3.75)\end{array}$ & $\begin{array}{c}2.16 \\
(1.40 \sim 3.34)\end{array}$ \\
\hline Sex & & $\begin{array}{c}1.73 \\
(1.16 \sim 2.60)\end{array}$ & $\begin{array}{c}1.75 \\
(1.17 \sim 2.64)\end{array}$ \\
\hline Age & & $\begin{array}{c}1.03 \\
(1.01 \sim 1.05)\end{array}$ & $\begin{array}{c}1.03 \\
(1.01 \sim 1.05)\end{array}$ \\
\hline Insurance & & $\begin{array}{c}0.82 \\
(0.23 \sim 2.89)\end{array}$ & $\begin{array}{c}0.86 \\
(0.24 \sim 3.03)\end{array}$ \\
\hline Treatment frequency & & & $\begin{array}{c}1.01 \\
(1.00 \sim 1.02)\end{array}$ \\
\hline Omnibus test & & $\begin{array}{c}x^{2}=20.33 \\
(\mathrm{p}<0.0001)\end{array}$ & $\begin{array}{c}x^{2}=5.33 \\
(\mathrm{p}<0.021)\end{array}$ \\
\hline Pseudo $R^{2}$ & 0.0332 & 0.0597 & 0.0666 \\
\hline
\end{tabular}

$\mathrm{N}=$ Numbers of case treated of Western/Korean Medicine after surgery

Table 8. Utilization of Medical Institution after Rotator Cuff surgery

\begin{tabular}{|c|c|c|c|c|}
\hline \multirow{3}{*}{ Variables } & \multicolumn{3}{|c|}{ Patients } & \multirow{3}{*}{ p-value } \\
\hline & ALL & Western Med & Korean Med & \\
\hline & $\mathrm{N}^{*}(\%)$ & $\mathrm{N}(\%)$ & $\mathrm{N}(\%)$ & \\
\hline \multicolumn{5}{|l|}{ Sex } \\
\hline Male & $536(50.66)$ & $493(52.84)$ & $43(34.4)$ & \multirow{3}{*}{0.0001} \\
\hline Female & $522(49.34)$ & $440(47.16)$ & $82(63.6)$ & \\
\hline Total & $1058(100)$ & $933(100)$ & $125(100)$ & \\
\hline \multicolumn{5}{|l|}{ Insurance } \\
\hline N.H.I* & $1,036(97.92)$ & 974(97.96) & $122(97.6)$ & \multirow{3}{*}{0.7374} \\
\hline M.A.P* \&V.H.C ${ }^{*}$ & $22(2.08)$ & $19(2.04)$ & $3(2.4)$ & \\
\hline \multirow[t]{2}{*}{ Total } & $1,058(100)$ & $933(100)$ & $125(100)$ & \\
\hline & Mean \pm SD & Mean \pm SD & Mean \pm SD & p-value ${ }^{*}$ \\
\hline Age & $54.94 \pm 11.91$ & $54.40 \pm 11.98$ & $58.96 \pm 10.53$ & $<0.0001$ \\
\hline Frequency of visites & $16.80 \pm 17.52$ & $15.99 \pm 17.24$ & $22.89 \pm 18.44$ & $<0.0001$ \\
\hline Total Medical Cost & $2,878,594 \pm 1,386,036$ & $2,833,612 \pm 1,372,968$ & $3,214,335 \pm 1,441,778$ & 0.0039 \\
\hline
\end{tabular}

${ }^{*} \mathrm{~N}=$ Numbers, $\mathrm{NHI}=$ National Health insurance, MAP = Medical Aid Program, VHC = Veterans' healthcare, $\mathrm{p}$-value calculated between Western Med group and Korean Med group 
health insurance, so there was no significant difference among the two groups.

Finally, the average number of treatments received by the WM group was 15.99 times and the total medical expense spent within 5 months averaged 2,833,612 KRW. And the average number of treatments received by the $\mathrm{KM}$ group was 22.89 times, and the total medical expense spent within 5 months was 3,214,335 KRW on average. Therefore, the KM group received treatments 6.9 more times on average, and spent 380,723 KRW more (Table 8).

\section{Medical service usage of $\mathrm{KM}$ and WM institutions depending on the treatment period of the rotator cuff surgery patients}

We compared the patients who completed their treatment within five months after the rotator cuff surgery (termination group) and the patients who either received a reoperation or were treated for more than five months after the surgery (continuation group). Out of the 1058 patients in total, the termination group was 517 patients $(48.87 \%)$ and the continuation group was 541 patients $(51.13 \%)$. Among the WM group, the termination group was 482 patients $(51.66 \%)$ and the continuation group was 451 patients (48.34\%). Among the KM group, the termination group was 35 patients $(28 \%)$ and the continuation group was 90 patients (72\%) (Table 9).

The frequency of treatments and the medical expenses were analyzed according to each group. Among the termination group, WM group used a WM institution 10.94 times on average, and the average medical expense was 2,623,825 KRW,

Table 9. Analysis of treatment results at each medical institution

\begin{tabular}{lcccc}
\hline \multirow{2}{*}{ Variables } & All & Western Med. & Korean Med. & \multirow{2}{*}{ p-value $^{*}$} \\
\cline { 2 - 4 } & $\mathrm{N}^{*}(\%)$ & $\mathrm{N}(\%)$ & $\mathrm{N}(\%)$ & $<0.0001$ \\
Treatment End $^{*}$ & $517(48.87)$ & $482(51.66)$ & $35(28)$ & $<0.0001$ \\
Treatment Continue $^{*}$ & $541(51.13)$ & $451(48.34)$ & $90(72)$ & \\
\hline
\end{tabular}

"Western Med.:Western Medical institution, Korean Med.:Korean Medical institution, p-value:p-value calculated between western med. and korean med., N:Number of persons, Treatment End:group whose treatment ends within 5 months, Treatment Continue:group whose treatment lasts more than 5 months

Table 10. Analysis of numbers of medical service users and cost of each institution after rotator cuff surgery

\begin{tabular}{|c|c|c|c|c|c|}
\hline Variables & & & $\mathrm{N}^{*}$ & Mean \pm SD & $(\operatorname{Min} \sim \operatorname{Max})$ \\
\hline \multirow{4}{*}{ Treatment End ${ }^{*}$} & \multirow{2}{*}{ Western Med } & Number of Visit & \multirow{2}{*}{482} & $10.94 \pm 10.94$ & $(1 \sim 78)$ \\
\hline & & Medical Cost & & $2.623 .825 \pm 1,158,574$ & $(8,310 \sim 13,470,180)$ \\
\hline & \multirow{2}{*}{ Korean Med } & Number of Visit & \multirow{2}{*}{35} & $16.17 \pm 9.98$ & $(3 \sim 35)$ \\
\hline & & Medical Cost & & $2,832,124 \pm 1,176,085$ & $(740,750 \sim 5,498,000)$ \\
\hline \multirow{4}{*}{ Treatment Continue ${ }^{*}$} & \multirow{2}{*}{ Western Med } & Number of Visit & \multirow{2}{*}{451} & $21.39 \pm 20.77$ & $(2 \sim 157)$ \\
\hline & & Medical Cost & & $3,057,820 \pm 1,540,079$ & $(93,270 \sim 16,783,190)$ \\
\hline & \multirow{2}{*}{ Korean Med } & Number of Visit & \multirow{2}{*}{90} & $25.5 \pm 20.27$ & $(3 \sim 100)$ \\
\hline & & Medical Cost & & $3,362,973 \pm 1,512,615$ & $(1,104,200 \sim 9,877,860)$ \\
\hline
\end{tabular}

${ }^{*} \mathrm{~N}=$ Persons, Treatment End $=$ End of treatment within 5 months, Treatment Continue = Treatment lasts more than 5 months 
while $\mathrm{KM}$ group used a $\mathrm{KM}$ institution 16.17 times on average, and the average medical expense was 2,832,124 KRW. Compared to the WM group, medical institutions were used 15.3 more times in the KM group, and medical expenses were 208,299 KRW higher.

Among the continuation group, WM group used a WM institution 21.39 times on average, and the average medical expense was 3,057,820 $\mathrm{KRW}$, while $\mathrm{KM}$ group used a $\mathrm{KM}$ institution 25.5 times on average, and the average medical expense was $3,362,973 \mathrm{KRW}$. Compared to the WM group, medical institutions were used 4.11 more times in the KM group, and medical expenses were $305,153 \mathrm{KRW}$ higher.

Within the WM group, the number of visits to a medical institution was 1.96 times higher and the medical expenses were about 1.17 times higher in the continuation group compared to the termination group. Within the KM group, the number of visits to a medical institution was 1.58 times higher and the medical expenses were about 1.19 times higher in the continuation group compared to the termination group. (Table $10)$.

\section{Discussion}

Utilizing the patient sample data from the Health Insurance Review \& Assessment Service, this study conducted analyses on insurance statements of rotator cuff tear patients, principal diagnosis according to surgery status, and patients' medical service usage behavior of KM and WM treatments. Following are the analysis results. N0936 was the most common (50.57\%) principal diagnosis to be operated on. Women were significantly more likely than men to receive $\mathrm{KM}$ treatments. About $50 \%$ of the patients start receiving KM treatments 73 days after the surgery. And finally, logistic regression analysis showed that treatment continuation group tended to receive $\mathrm{KM}$ treatments.

According to an analysis of the frequency of principal diagnosis of patients who received surgery, N0936 had the highest percentage (50.57\%) of rotator cuff surgery.

We conducted a six-month follow-up analysis of patients who underwent rotator cuff surgery before June 1, 2015 and studying the percentile of the start date of $\mathrm{KM}$ treatment found that around $50 \%$ of median patients start $\mathrm{KM}$ treatments 73 days after the operation. In WM institutions, postoperative rehabilitation includes early manual exercises to prevent adhesion and rigidity and starts active and isotonic exercises around 6 8 weeks after the surgery (21). Although the patients remain hospitalized after the surgery for a designated rehabilitative period, in many cases WM rehabilitative therapy cannot be carried out effectively due to severe postoperative pain (22). And we may infer that the patients start KM treatments later on, after they become dissatisfied with WM rehabilitative therapy. We conducted a logistic regression analysis on the treatment continuation group to study the impact of the late start date of KM treatment. Model 1's OR was 2.75, Model 2's OR was 2.47, and Model 3's OR was 2.16, which confirmed that out of the postoperative treatment groups, the continuation group was more likely to receive KM treatments. As a result, we may infer that 
the demand for KM treatments increases over time after rotator cuff surgery.

In addition, there was no significant difference between men (52.84\%) and women (47.16\%) among postoperative patients, while women (63.6\%) were more likely than men $(34.4 \%)$ to receive KM treatments postoperatively. Further research is needed to find out whether this is due to a difference in occupational characteristics because the time required for $\mathrm{KM}$ is longer than WM (13).

In addition, the number of medical service usage of postoperative KM patients (22.89 times on average) is higher than that of postoperative WM patients (15.99 times on average). We speculate this may be because the late start date of $\mathrm{KM}$ treatments (22) due to ineffective WM postoperative rehabilitation therapy increased the possibility of rotator cuff adhesion and rigidity of subjects (22). However, this study was not able to distinguish between patients who solely receive KM treatments versus those who received both $\mathrm{KM}$ and $\mathrm{WM}$ treatments due to essential pre- and postoperative treatments in WM institutions. Therefore, it is necessary to conduct further study on the patient group who received sole KM treatment after the surgery.

Analyzing the data of all patients registered on HIRA_NPS in 2015, the number of WM treatments in the rotator cuff surgery group (approximately 43.40) was higher than that of the total population excluding the target group (approximately 16.62). And the number of KM treatments in the rotator cuff surgery group (approximately 5.26) was also higher than that of the total population excluding the target group (approximately 2.28).

Based on the insurance claims, the average cost for rotator cuff surgery in 2015 was 2,474,634 KRW on average. Comparing the medical expenses in 2015 of rotator cuff surgery group versus the rest of the population, the target group spent a significantly higher amount $(4,626,323 \mathrm{KRW}$ on average) than the total population excluding the target group $(1,033,411 \mathrm{KRW}$ on average). The expense seems to be higher because it includes the cost of surgery, but even excluding the cost of surgery, it appears to be higher due to the pre- and postoperative treatment procedures. This can be seen as raising the possibility that the overall cost of rehabilitation treatment can be reduced if a rapid transition to $\mathrm{KM}$ rehabilitation treatment is made when symptom improvement of Western rehabilitation treatment is not easily achieved. Therefore, it appears that additional systematic studies are needed on the effectiveness of $\mathrm{KM}$ rehabilitation treatment when symptoms improve after rotator cuff tear surgery.

Looking at rotator cuff surgery patients as the subject of this study, from 1980 to 2005, the number of acromioplasty increased six times from 3.3 to 19 per 10,000 patients (23). Also, M75 (shoulder lesion), which is a high frequency principal diagnosis for rotator cuff tear, ranked 32nd (24), 29th (25), and 29th (26) in terms of WM insurance payment per disease classification in 2013, 2014, and 2015, showing a high utility of WM medical service. Furthermore, M75 ranked as 4th (27), 5th (28), 5th (29) in the KM insurance payment per disease classification for the same years ranked, showing even higher ranks than the WM list. 
However, it is not possible to conclude that the demand for $\mathrm{KM}$ is absolutely high because the number of postoperative $\mathrm{KM}$ use by patients (22.89 times on average) is higher than the number of postoperative WM use (15.99 times on average), and further research is needed.

This study has following limitations in terms of research methodology. First, there is insufficient data on the number of days that each treatment group visited a medical institution. And because we do not know whether the patients received collaborative treatment or separate treatment by each treatment group, the exact content of medical practice is unknown, and the analysis criteria is ambiguous. Further research is likely to be possible when the recently discussed pilot project on KM-WM collaborative project is completed with additional data on the number of hospital visit days, the interval between visits and the set-up of collaborative treatment criteria.

Second, the 2015 Health Insurance Review and Assessment Service patient sample data is limited in that it is a sample, and not complete census data. It does not include statistical data on the many types of uninsured treatment practices that are widely used in $\mathrm{KM}$ and $\mathrm{WM}$ institutions, so we were not able to analyze all the medical treatments that are currently being practiced in the real world.

Third, since this study is conducted with sample data only from 2015, the data for continuous treatment that goes beyond one year may be omitted, resulting in deviation. To eliminate this potential deviation, we may exclude data of patients from the first and last three months of the year. However, reducing the total amount of data available may result in other types of deviations.

Fourth, in this study, we tried to investigate detailed information about the patient's rotator cuff tear status as much as possible by analyzing all the KCD injuries applied to each ICD-10-PCD. However, because Grade of rotator cuff tear(30) cannot be applied on coding system, there is difficulty to investigate a appropriate $\mathrm{KM}$ rehabilitation treatment on each grade. Therefore it is necessary to additionally analyze the $\mathrm{KM}$ rehabilitation treatment according to the image diagnosis of the actual patient.

Finally, KM and/or WM practitioners may not write down the principal diagnoses and treatment practices according to accurate standards, which may lead to difficulties in distinguishing the principal diagnoses and practices that accurately correspond to rotator cuff surgery. To improve the accuracy, further studies will be required to obtain error rates through case studies of actual treatments

\section{Conclusion}

As a result of analyzing the $\mathrm{KM}$ and $\mathrm{WM}$ institution usage behavior of patients with rotator cuff tear via 2015 sample data from the Health Insurance Review \& Assessment Service, the number of visits to a medical institution and medical expenses were higher for the patients who continue to receive treatments five months after the surgery. And further observation and future research are needed for more effective treatments and reduced medical costs. 


\section{Thank To}

This study is the master's thesis of Gachon University in 2018.

\section{References}

1. S.Brent B, Roberts CM Manske. (2012). Clinical orthopaedic rehabilitation: an evidence -based Approach-Third edition. The Journal of the Canadian Chiropractic Association. 56(3), 234.

2. Kim YK. (2008). Controversy in pathophysiology of rotator cuff tear: degenerative Tear. Journal of the Korean Shoulder and Elbow Society. 11(2), 71-6. https://doi.org/10.5397/CiSE.2008. 11.2.071

3. Kim SY, Chae YB, Lee HS, Ko YM, Lee HJ, Park HJ. (2012). Study for clinicians' perception on the acupuncture research and ways to symbiosis between basic research and clinical practice. Korean Journal of Acupuncture. 29(2), 224-38.

4. Neviaser RJ, Neviaser TJ. (1990). Observations on impingement. Clinical orthopaedics and related research. 25(4):60-3.

5. DeOrio J, Cofield RH. (1987). Results of a second attempt at surgical repair of a failed initial rotator-cuff repair. The Journal of bone and joint surgery American volume. 66(4), 563-7.

6. Shin SJ, Chung J, Lee J, Ko YW. (2016). Recovery of muscle strength after intact arthroscopic rotator cuff repair according to preoperative rotator cuff tear size. The American journal of sports medicine. 44(4),

\section{2-80. $10.1177 / 0363546515625043$}

7. Lee KJ, Park CH, Lee YJ, Lee JH, Cho JH, Park TY, et al. (2017). Trends of Korean medicine treatment after musculoskeletal disorder surgery: a literatural review. Journal of Korean Medicine Rehabilitation. 27(3), 61-70. https://doi.org/10.18325/jkmr.2017.27. 3.61

8. Kim TR, Kim YJ, Woo CH. (2017). 45 Cases Report of Korean Medicine Rehabilitation after Surgery. Journal of Korean Medicine Rehabilitation. 27(1), 83-9. https://doi.org/10. 18325/jkmr.2017.27.1.83

9. Lee JE, Oh MS. (2015). The retrospective review of 410 shoulder pain inpatients in Korean medicine hospital. Journal of Korean Medicine Rehabilitation. 25(2), 155-73. https://doi.org/10.18325/jkmr.2015.25.2.155

10. Kwon MG, Jo HG, Kim JH, Jeung CW, Go YJ, Seol JU, et al. (2017). The analysis of east-west integrative care system in a Korean medicine hospital using by EMR data: preliminary study. Journal of Korean Medicine Rehabilitation. 27(2), 93-9. https://doi.org/10. 18325/jkmr.2017.27.2.93

11. Jin HJ, Baek Y, Kim HS, Ryu J, Lee S. (2015). Constitutional multicenter bank linked to Sasang constitutional phenotypic data. BMC complementary and alternative medicine. 15(1), 1-8. 10.1186/s12906-015-0553-3

12. Korean Medicine Data Center(KDC) [Available from: https://kdc.kiom.re.kr/html/.

13. Park HS, Uhm TW, Kim NK. (2017). A study on the facial palsy patients' use of Western-Korean collaborative treatment: Using Health Insurance Review \& Assessment 
Service-National Patients Sample. Journal of the Korean Data and Information Science Society. 28(1), 75-86. https://doi.org/10.7465/ jkdi.2017.28.1.75

14. Kim D, Lim B. (2017). The trend of Korean Medicine utilization in 2008-2013. Journal of Society of Preventive Korean Medicine. 21(1), 57-66.

15. Uhm T, Kim NK, Kim S. (2015). An analysis on present condition of the cooperative medical care using the episode of care: claims data of health insurance review \& assessment service. Journal of Society of Preventive Korean Medicine. 19(2), 51-6.

16. Wu MS, Chen KH, Chen IF, Huang SK, Tzeng PC, Yeh ML, et al. (2015). The efficacy of acupuncture in postoperative pain management: a systematic review and meta -analysis. PloS one. 11(3), 9. https://doi.org/ 10.1371/journal.pone.0150367

17. Song MY, Jo HG, Sul JU, Leem J. (2018). Proposal of East-west Integrative Medicine manual for Rehabilitation after shoulder surgery. Journal of Korean Medicine Rehabilitation. 28(1), 109-20. https://doi.org/ 10.18325/jkmr.2018.28.1.109

18. Lee GE, Kim YI, Jo KS, Han SH, Kim MK, Min BK, et al. (2018). Forty-One Cases of Rotator Cuff Injuries Treated by Complex Korean Medicine Treatment: A Retrospective Review. Journal of Korean Medicine Rehabilitation. 28(4), 81-7. https://doi.org/10. 18325/jkmr.2018.28.4.81

19. Korea Ministry of Government Legislation [Available from: http://www.law.go.kr.

20. Lee SM, Hwnag JH, Kim DW. (2014).
Osteolysis after arthroscopic rotator cuff repair using absorbable suture anchor. Journal of Korean Shoulder and Elbow Society. 7-7.

21. Seo JB, Bahng SC. (2016). Early complications after repair of massive rotator cuff tear. Clinics in Shoulder and Elbow. 9(1), 27-33. https://doi.org/10.5397/CiSE.2006.9.1.027

22. Kim YJ, Won JH, Ahn HD, Woo CH. (2014). A Clinical Case of Korean Rehabilitation Treatment for Rotator Cuff Tear. The journal of east-west medicine. 39(3), 30-9.

23. Yu E, Cil A, Harmsen WS, Schleck C, Sperling JW, Cofield RH. (2010). Arthroscopy and the dramatic increase in frequency of anterior acromioplasty from 1980 to 2005: an epidemiologic study. Arthroscopy: The Journal of Arthroscopic \& Related Surgery. 26(9), S142-S7. https;//doi.org/10.1016/j.arthro.2010. 02.029

24. KOSIS. The Status of the Payment of the Multiple Cords by the Classification of Diseases(2013) 2013 [Available from: https://kosis.kr/statHtml/statHtml.do?orgId=35 0\&tblId=DT_35001_A070111\&vw_cd=MT_Z TITLE\&list_id=350_35001_6\&seqNo=\&lang _mode=ko\&language $=$ kor\&obj_var_id=\&itm id $=\&$ conn_path $=$ MT_ZTITLE.

25. KOSIS. The Status of the Payment of the Multiple Cords by the Classification of Diseases (2014) 2014 [Available from: https://kosis.kr/ statHtml/statHtml.do?orgId=350\&tblId=DT_3 5001 A071111\&vw cd=MT ZTITLE\&list id $=350 \_35001 \_6 \&$ seqNo $=\& l a n g \_$mode $=$ko\&lan guage $=$ kor\&obj_var_id $=\& \mathrm{itm} \_\mathrm{id}=\&$ conn_path =MT_ZTITLE.

26. KOSIS. The Status of the Payment of the 
Multiple Cords by the Classification of Diseases(2015) 2015 [Available from: https://kosis.kr/statHtml/statHtml.do?orgId=35 0\&tblId=DT_35001_A072111\&vw_cd=MT_Z TITLE\&list_id=350_35001_6\&seqNo=\&lang _mode $=$ ko\&language $=$ kor\&obj_var_id=\&itm id=\&conn_path=MT_ZTITLE.

27. KOSIS. The Status of the Payment of the Multiple Cords by the Classification of Diseases (2013_Korean Medicine) 2013 [Available from: https://kosis.kr/statHtml/statHtml.do?orgId= 350\&tblId=DT_35001_A80211\&vw_cd=MT_ ZTITLE\&list_id=350_35001_6\&seqNo=\&lan g_mode $=$ ko\&language $=$ kor\&obj_var_id $=\& i t m$ _id=\&conn_path=MT_ZTITLE.

28. KOSIS. The Status of the Payment of the Multiple Cords by the Classification of Diseases (2014_Korean Medicine) 2014 [Available from: https://kosis.kr/statHtml/statHtml.do?orgId= 350\&tblId=DT_35001_A81211\&vw_cd=MT_ ZTITLE\&list_id=350_35001_6\&seqNo=\&lan g_mode $=$ ko\&language $=$ kor\&obj_var_id $=\& i t m$ _id=\&conn_path=MT_ZTITLE.
29. KOSIS. The Status of the Payment of the Multiple Cords by the Classification of Diseases(2015_Korean Medicine) 2015 [Available from: https://kosis.kr/statHtml/statHtml.do? orgId=350\&tblId=DT_35001_A82211\&vw_c $\mathrm{d}=\mathrm{MT}$ ZZTITLE\&list_id=350_35001_6\&seqN $\mathrm{o}=$ \&lang_mode $=$ ko\&language $=$ kor\&obj_var_i $\mathrm{d}=\& \mathrm{itm} \_$id $=\&$ conn_path=MT_ZTITLE.

30. SJ Shin, MJ Seo. (2014). Partial Thickness Rotator Cuff Tears. Clinics in Shoulder and Elbow. 17(2). 91-100. https://doi.org/10.5397/ CiSE.2014.17.2.91

\section{ORCID}

Hyun-jin Khang https://orcid.org/0000-0002-6504-7331 Hye-Yoon Lee https://orcid.org/0000-0002-9486-1703 Se-Yeon Lee https://orcid.org/0000-0003-4648-228X NamKwen Kim https://orcid.org/0000-0001-6969-1231 Yun Kyung Song https://orcid.org/0000-0002-7666-6832 